\title{
APPLICABILITY OF RESONANT TWO PHOTON IONIZATION IN SUPERSONIC BEAMS TO HALOGENATED AROMATIC HYDROCARBONS
}

R. Tembreull and D. M. Lubman

University of Michigan, Ann Arbor, MI 48109

\section{ABSTRACT}

This work investigates problems encountered in the application of one color resonant two-photon ionization as an ionization source in supersonic beam mass spectroscopy. of particular interest is the fact that photons of one color may not provide sufficient energy to cause ionization even when the laser source is tuned to an excited vibronic molecular state. We have therefore correlated trends in ionization potential with molecular structure for simple systems, specifically, halogenated aniline, phenol and toluene derivatives and mono- and di-substituted benzenes. In the case of para-substituted compounds where there is little substituent group interaction ionization occurs efficiently at the $\mathrm{s}_{\rightarrow} \rightarrow \mathrm{S}_{1}$ origin, provided ultrafast processes are not active as in the case of iodo substituted benzenes. Many ortho compounds, however, are found not to ionize efficiently. This is probably due to a combination of coulombic and steric interactions which result in an increase in ionization potential. These types of effects have been qualitatively related to the electron releasing and withdrawing properties of the substituent groups thereby allowing reasonable predictions to be made regarding those types of substituted benzenes which can be probed with R2PI.

\section{INTRODUCTION}

With the advent of coherent lasers it is now possible to routinely study processes which involve multiple photon excitation. One such process, resonant two photon ionization (R2PI) has significant analytical potential as a wavelength selective ionization source in mass spectrometry. We have therefore addressed the question of the general applicability of R2PI as an analytical tool in this work.

R2PI involves the absorption of two photons by a molecule and results in the production of a positive ion and concurrent release of an electron. Absorption of the first photon is strongly enhanced when the frequency of the ionizing laser is tuned to an excited vibronic state. As a result a large ionization signal is observed when the laser is in resonance with such an intermediate state, e.g. a vibronic $\pi^{*}$ state in an aromatic molecule. Absorption of the second photon, on the other hand, is into an ionization continuum where the cross section for absorption is not a strong function of wavelength. Consequently, R2PI spectra are in effect absorption spectra. R2PI therefore provides wavelength selectivity thereby adding an extra dimension 
to conventional analytical mass spectrometry. R2PI is also characterized by excellent sensitivity allowing detection below ppb levels. Further, R2PI provides an extremely efficient means of "softly" ionizing molecules for mass spectroscopic detection. Thus it is possible to obtain the molecular weight of fragile biological or inorganic molecules which tend to fragment in electron impact ionization.

In order to fully exploit the wavelength dependence and spectroscopic information available from R2PI we have coupled our time-of-flight mass spectrometer (TOFMS) with a supersonic jet source in order to provide ultracold molecules. This is significant because at room temperature ultraviolet absorption spectra are broad and often featureless in contour due to the population of a large manifold of rovibrational states in the ground electronic state. Hence, wavelength selectivity is difficult or impossible to achieve. However, with the use of supersonic beam methodology, sharp rovibronic bands are observed which are typically $\sim 2 \mathrm{~cm}^{-1}$ in width. As a result individual components can be identified in complex mixtures even when the analyte is present at a very low concentration.

Analytically a major drawback of R2PI supersonic beam mass spectrometry is the meager and often conflicting data base available. For example, many molecular ionization potentials are unavailable in the literature and those which are avajlable are frequently in error. Such knowledge is crucial in this technique because in order to observe a resonant ionization signal, the sum of the energy of the two photons absorbed by a molecule must exceed its ionization potential. Therefore in this study the ability to make reasonable predictions regarding the effect various substituents have on the energy states involved in the resonant ionization of simple benzenoid systems is investigated. Trends in ionization potential are correlated with the type and position of substituent groups on a benzene ring.

\section{EXPERIMENTAL}

Our experimental apparatus consists of a six port vacuum cross in which a laser beam, the supersonic jet and a time of flight mass spectrometer are all mutually perpendicular. The supersonic molecular beam is generated by a pulsed valve which reduces the duty cycle of the gas flow compared to a continuous flow so that a small 6" pumping station can be used to maintain pressures at $5 \times 10^{-6}$ torr. This molecular beam modulator allows the use of a large expansion orifice $(0.5 \mathrm{~mm})$ which can provide high $\left(\sim 10^{15}\right.$ molecules/cc) on-axis intensity in the free jet. In turn, high sensitivity can be obtained in our measurements relative to present continuous jet expansions which require an orifice $100 \mathrm{x}$ smaller to maintain similar pumping conditions. The ions are created in the jet by tunable UV radiation $(\sim 1-3 \mathrm{~mJ})$ generated by frequency doubling the output of a tunable Nd:YAG pumped dye laser system. The ions are then mass analyzed in a 
time-of-flight mass spectrometer in which resolution $>1000$ can be obtained and the spectroscopy of a particular mass peak is monitored by a gated integrator in order to obtain "mass selected" ionization spectra.

\section{RESULTS AND DISCUSSION}

Three states are involved in R2PI. For the substituted benzenes these states are the $\pi$ electronic ground state, $S$, the intermediate $\pi^{*}$ state, $S_{1}$, and the jonic ground electronic state, $s^{*}$. As pointed out by previous investigators the effect of a substituent on the intermediate $\pi^{*}$ state of benzene is similar to its effect on the ground ionic state. This is reasonable since in the intermediate state an electron has been promoted from the stable $4 n+2$ aromatic $\pi$ bonding core structure to a $\pi^{*}$ antibonding orbital creating a "photohole", whereas in the ground ionic state an electron has been removed altogether from the benzene ring. Release of electron density to or removal of electron density from the ring system therefore affects the energy of these states in a similar manner. Electron releasing groups such as $-\mathrm{CH}_{3},-\mathrm{OH}$, and -NH stabilize these upper states. This is reflected in the absorption spectra and PES of these compounds, both of which exhibit a red shift dependent on the ability of the substituent to release electron density to the ring system. The converse is true for electron withdrawing groups. Significantly, substituents which interact strongly with the aromatic $\pi$ system do not exhibit linearly additive effects and are difficult to characterize theoretically. We have therefore looked for experimental trends upon which a simple model may be based.

The first class of molecules studied were the monosubstituted benzenes. The shift to lower energy of both the $S_{A} \rightarrow S_{1}$ absorption and the ionization potential of aniline, phenol and toluene are easily rationalized in terms of the overall dipole properties of each species. A simple electrostatic dipole model predicts that both the "permanent dipole" associated with each substituent as well as the dipole induced in the substituent by the presence of charge on the ring center favor release of electron density to the ring. For the monohalogenated benzenes however, the magnitude of the induced dipole is comparable to that of the permanent dipole and opposite in direction. Thus, halogen substituents may be either electron releasing or electron withdrawing depending on what other substituents are also present on the ring. In all the cases investigated herein the halogens were found to act as electron releasing substituents towards the aromatic $\pi$ system.

The disubstituted benzenes proved to be quite interesting. When a strong electron releasing group, such as $-\mathrm{OH}$ or $-\mathrm{NH}_{2}$, is "ortho" to a halogen substituent two photons of one color are either insufficient to cause ionization or resonant ionization is very weak. Several ortho dihalogenated compounds were also found not to exhibit any detectable spectrum well to the blue of 
their electronic origins, e.g. o-dibromobenzene and o-bromochlorobenzene. These effects are believed to be due to coulombic interaction between adjacent groups which are particularly effective in stabilizing the $S_{0}$ and $S_{1}$ states relative to ${ }^{0}$ the ionic ground electronic state $\mathrm{s}^{*}$. This results in an increase in the energy gap between the $s$, and $S^{*}$ states. Thus, not only is ionization usually not observed at the $0 \rightarrow 0$ origin, but furthermore, when two photons of one color are sufficient to cause resonant ionization they must proceed through a highly excited vibrational level in the $S_{1}$ state. Poor Franck-Condon factors therefore result in signals of small intensity. These types of effects were not observed for halogenated benzenes containing a moderately electron releasing group as Fig. 1 illustrates. It is also noteworthy that electronic transitions other than $\pi \pi^{*}$ transitions may be probed for some compounds as has been demonstrated for $n \pi^{*}$ transitions 2 in several azabenzenes. ${ }^{2}$

1. R. Tembreull \& D.M. Lubman, Anal. Chem., 56, 1962 (1984).

2. R. Tembreul1, C.H. Sin, H.M. Pang \& D.M. Lubman, Anal. Chem., 5i, 2911 (1985).

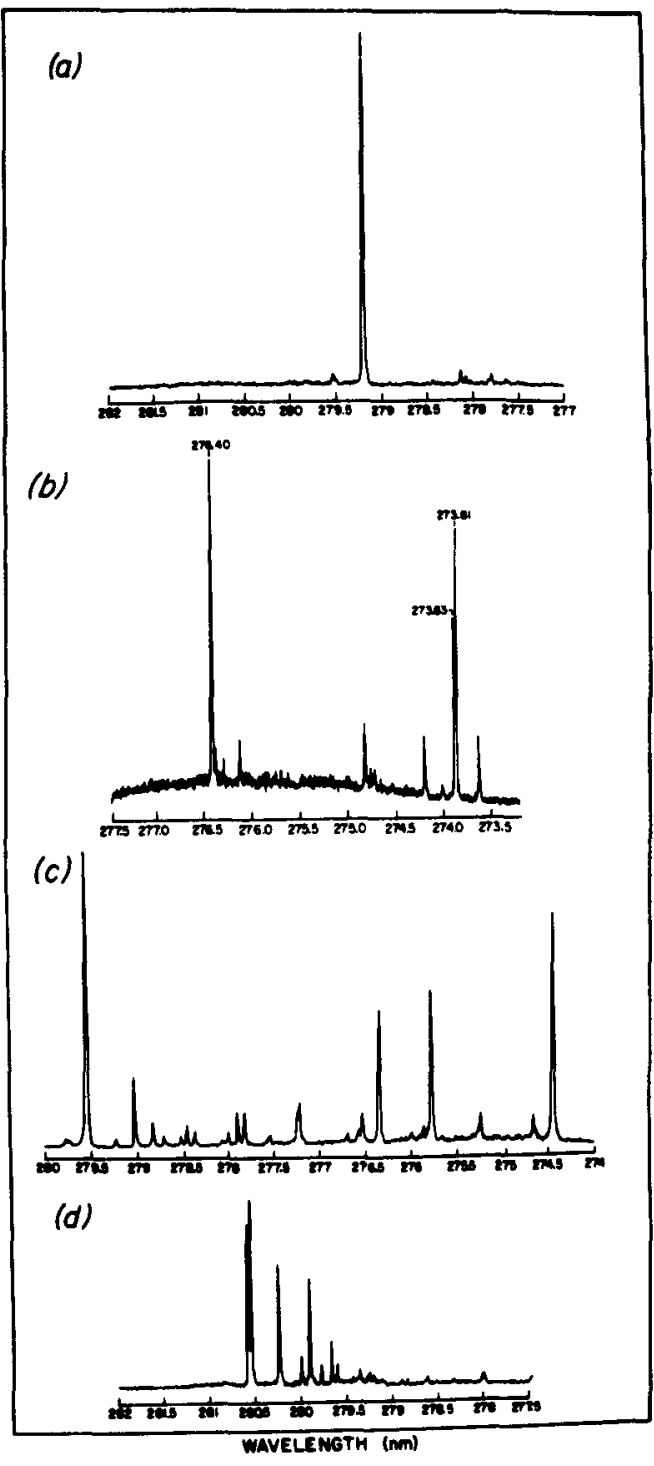

Fig. 1. Resonant two-photon ionization spectra taken in an expansion of 1 atm back pressure of Ar of (a) 2,4-dichlorotoluene, (b) 2,6-dichlorotoluene, (c) $2,5-$ dichlorotoluene, and (d) 3,4dichlorotoluene. Only the molecular ion is monitored in these spectra using a TOF mass spectrometer. 